\title{
A bibliometric analysis of the dental scientific literature on COVID-19
}

\author{
Jelena Jacimovic ${ }^{1}$ (D) - Aleksandar Jakovljevic ${ }^{2}$ (D) $\cdot$ Venkateshbabu Nagendrababu $^{3}$ (D) $\cdot$ Henry Fergus Duncan ${ }^{4}$ (D) \\ Paul M. H. Dummer ${ }^{5}$
}

Received: 18 December 2020 / Accepted: 25 March 2021 / Published online: 6 April 2021

(C) The Author(s), under exclusive licence to Springer-Verlag GmbH Germany, part of Springer Nature 2021

\begin{abstract}
Objectives The rapid production of a large volume of literature during the early phase of the COVID-19 outbreak created a substantial burden for clinicians and scientists. Therefore, this manuscript aims to identify and describe the scientific literature addressing COVID-19 from a dental research perspective, in terms of the manuscript origin, research domain, study type, and level of evidence (LoE).

Materials and methods Data were retrieved from Web of Science, Scopus, and PubMed. A descriptive analysis of bibliographic data, collaboration network, and keyword co-occurrence analysis were performed. Articles were further classified according to the field of interest, main research question, type of study, and LoE.

Results The present study identified 296 dental scientific COVID-19 original papers, published in 89 journals, and co-authored by 1331 individuals affiliated with 429 institutions from 53 countries. Although $81.4 \%$ were single-country papers, extensive collaboration among the institutions of single countries (Italian, British, and Brazilian institutions) was observed. The main research areas were as follows: the potential use of saliva and other oral fluids as promising samples for COVID-19 testing, dental education, and guidelines for the prevention of COVID-19 transmission in dental practice. The majority of articles were narrative reviews, cross-sectional studies, and short communications. The overall LoE in the analyzed dental literature was low, with only two systematic reviews with the highest LoE I.

Conclusion The dental literature on the COVID-19 pandemic does not provide data relevant to the evidence-based decisionmaking process. Future studies with a high LoE are essential to gain precise knowledge on COVID-19 infection within the various fields of Dentistry.

Clinical relevance The published dental literature on COVID-19 consists principally of articles with a low level of scientific evidence which do not provide sufficient reliable high-quality evidence that is essential for decision making in clinical dental practice.
\end{abstract}

Keywords COVID-19 $\cdot$ SARS-CoV-2 $\cdot$ Epidemics $\cdot$ Dentistry $\cdot$ Bibliometrics $\cdot$ Knowledge discovery

Aleksandar Jakovljevic

dr.sasuli@hotmail.com

Jelena Jacimovic

jelena.jacimovic@stomf.bg.ac.rs

Venkateshbabu Nagendrababu

hivenkateshbabu@yahoo.com

Henry Fergus Duncan

Hal.duncan@dental.tcd.ie

Paul M. H. Dummer

Dummer@cardiff.ac.uk
Central Library, School of Dental Medicine, University of Belgrade, Belgrade, Serbia

2 Department of Pathophysiology, School of Dental Medicine, University of Belgrade, Belgrade, Serbia

3 Department of Preventive and Restorative Dentistry, College of Dental Medicine, University of Sharjah, Sharjah, UAE

4 Division of Restorative Dentistry and Periodontology, Dublin Dental University Hospital, Trinity College Dublin, Dublin, Ireland

5 School of Dentistry, College of Biomedical and Life Sciences, Cardiff University, Cardiff, UK 


\section{Introduction}

The continuing outbreak of COVID-19 has raised serious concerns among the medical and research communities who have been faced with a highly dynamic and intensifying disease landscape. Although this new pathological entity is of exceptional virulence, the scientific community has struggled with insufficient information, inexperience, and a lack of supporting study data, and as a result, there has been an enormous demand to rapidly produce COVID-19-related evidence that can provide a basis for effective clinical decision-making and patient management.

The dental community has also embraced the commitment to focus research in the area in order to produce answers to a wide range of critical questions regarding COVID-19. Similar to General Medicine, leading journals from the field of Dentistry, Oral Surgery, and Oral Medicine have published a substantial number of articles aimed at guiding clinicians in the best management of patients with COVID-19 and to deliver expedient solutions to crucial questions in a short time frame. The dental literature on COVID-19-related issues includes the following: epidemiological aspects, patient treatment, protection of healthcare professionals, organization of hospital facilities, therapeutic efficacy of drugs, and identifying at-risk populations, all of which can assist healthcare providers and policymakers to make evidence-based decisions and informed recommendations for both treating patients and minimizing its transmission within dental clinics and staff.

The unprecedented generation of a large volume of scientific data and countless reports in a short time frame, providing recommendations for more reliable COVID-19 prevention and management, have shaped critical health communications and global pandemic discourse. The race to publish, an already known scientific phenomenon, has considerably expanded, impeding scientific advancement and raising concerns about the scientific publication process, potentially being compromised in many aspects due to the emergency situation. Further concerns are related to the inflation of publication metrics, used by journals that publish articles of questionable scientific value on popular topics [1]. That said, the production of a large volume of literature quickly during the early outbreak created a substantial burden for scientists and clinicians to critically evaluate the burgeoning output to discover research that delivered novel high-quality information, rather than those outputs summarizing or commenting on existing knowledge [2]. Furthermore, the rapid production of data introduces a potential concern over the accuracy, quality, postpublication review, and critical assessment of the COVID-19related scientific literature, which has led to several corrections and retractions, principally related to issues and errors in the results or data presented [3].

Recently, bibliometric analysis has become an accepted approach to present the current status and research patterns of a specific scientific domain; obtain information on the progress of particular knowledge; or highlight the most relevant sources, authors, institutions, and countries involved in the area. Several bibliometric studies were conducted during the previous months of the pandemic to explore the activity and trends of COVID-19 research in the medical field [4-6]. Insightful data on the extent, type, and level of evidence of COVID-19 scientific output and identification of wellresourced areas of study in Dentistry provides a valuable reference to original research available for decision-making and clinical treatment support, as well as to direct future research in this area. Based on this knowledge, editors, peer reviewers, and publishers can develop or redefine journal management strategies to provide priority space for submissions addressing crucial COVID-19 issues. As a result, an in-depth analysis of the scope and nature of scientific articles on COVID-19 in Dentistry, Oral Surgery, and Oral Medicine field needs to be conducted. Therefore, this paper aims to identify and analyze the characteristics (demographic data, study origin, study type) of scientific literature addressing COVID-19 from a dental research perspective, to recognize the most prolific authors, institutions, and countries of origin, prior to investigating dental research trends and publishing patterns related to the current pandemic.

\section{Materials and methods}

The main objective of this paper was to conduct a bibliometric analysis of articles related to COVID-19 and published in the dental research field. To offer the academic community a general picture of the way dental researchers have faced the pandemic, this bibliometric analysis concentrated on the COVID-19 dental scientific literature, examining its volume, authorship (individuals, institutions, and countries), publication sources (journals, conference proceedings, etc.), achieved impact according to the number of citations, and its content, considering the fields of interest, study designs, research questions, and level of evidence most commonly related to COVID-19 manuscripts.

The selection criteria of this bibliometric analysis included original scientific articles, case reports, review articles, and short communications published in the research field Dentistry, Oral Surgery, and Medicine (dental, scientific, or multidisciplinary journals) that contained terminology or keywords related to COVID-19 (including but not limited to SARS-CoV-2, COVID, novel coronavirus 2019) in the title, abstract, or keywords. Editorials, letters, news, commentaries, perspectives, opinions, scientific blogs, or technical notes were excluded from this research. Besides, manuscripts related to other similar coronaviruses, such as MERS-CoV or SARS-CoV, if SARS-CoV-2 or synonyms were not 
mentioned in the title, abstract, or keywords, as well as papers unavailable in full-text format, were also excluded.

\section{Data sources and search strategy}

Data for this research were retrieved from Clarivate Analytics' Web of Science (including Web of Science Core Collection (WoS), Korean Journal Database (KJD), Russian Science Citation Index (RSCI), SciELO Citation Index (SCIELO)), Scopus, and PubMed, from inception to September 6, 2020, and without language restrictions. Two independent investigators (J.J. and A.J.) conducted preliminary searches of these databases to ensure the validity of the proposed concept, avoid duplication of previously considered issues, develop the most optimal information retrieval strategy, and identify relevant COVID-19 publications. Test search strategies were also used to verify the performance of selected free keywords, synonyms, and appropriate subject indexing (e.g. Medical Subject Headings (MeSH), https://www.ncbi.nlm.nih.gov/ mesh), related to COVID-19 as the principal research concept. The final search strategy was customized according to the characteristics of each specific database to obtain as many eligible results as possible. Full details on the literature search strategy can be found in Online Resource 1.

\section{Study selection and data extraction}

A complete metadata report was exported in plain text or BibTeX format from the searched databases and imported into the $\mathrm{R}$ environment for statistical computing and graphics [7] and duplicates removal. The selection of studies included in this analysis was undertaken by two independent investigators (A.J. and J.J.) who screened titles, abstracts, and full texts of all articles identified in the electronic literature search. With the investigators' joint agreement, articles irrelevant to the defined research questions were excluded from the analysis.

The existing bibliographic attributes of all selected articles, such as authors' names and affiliations, year of publication, document title, abstract, publication name, document type, language, citation count, keywords, or DOI, were automatically extracted by the principal investigator (J.J.) and saved in a specially created spreadsheet. In addition, the impact factor (IF) of the journal based on the current release of Journal Citation Reports (JCRs) and Journal IF Quartile in the category Dentistry, Oral Surgery, and Medicine (based on the Clarivate JCRs 2019) were also recorded. Each article was further reviewed by two investigators (A.J. and J.J.), who independently classified identified papers according to a field of interest, main research domain, type of study, and level of evidence (LoE) as previously described [8]. Any disagreement between the two investigators was resolved by consensus and discussion with the third investigator (V.N.).

\section{Data analysis and visualization}

A descriptive analysis of bibliographic data focused on the previously formulated research questions, as well as network extraction, was completed using the bibliometrix R-package [9]. To disambiguate certain terms and to remove transcription or indexing errors, names of authors, institutions, and countries were also manually refined and normalized. All institutional affiliations were normalized and included on a macro level, such as universities and research centers, while microorganizations (i.e., individual departments or research units) were discarded. Articles originating from England, Scotland, and Wales were recategorized as being from the UK. Papers from Taiwan were included in the China category.

The main results of the bibliometric analysis describe the collection in terms of the number of papers, authors, institutions, countries, sources, keywords, and the number of citations. The total number of contributing authors and the frequency of their appearances were recorded in detail. The contribution of each author was assessed by applying the full and fractionalized counting method [10], giving each contributing author a score of one (e.g., three authors each receive one full credit) or a fraction of one credit (e.g., three authors receive one-third of a credit), respectively. Additionally, the contributions of authors were analyzed using dominance factor (DF) as a ratio indicating the fraction of multi-authored articles in which an author appears as the first author [11]. Citation counts for each evaluated paper were retrieved from the Clarivate Web of Science Core Collection (Times Cited Count). Collaboration analysis was used to identify coauthorships and determine collaboration networks of authors, institutions, or countries [12], laid out using the KamadaKawai algorithm [13] and the Louvain clustering method for detecting communities in networks [14]. Keyword cooccurrence analysis was done to map and cluster terms extracted from keywords of the analyzed collection. In addition to keyword analysis, the distribution of articles based on the field of interest, research domain, study design, and LoE was used to identify and describe COVID-19-related dental research topics and their strength based on observed levels of scientific evidence. Online Resource 2 presents the basis for the categorization of the articles into different LoEs. No LoE was assigned to animal, in vitro, laboratory, pilot, and simulation studies, as well as short communications. Further, within the pyramid of evidence employed, the level of scientific evidence of systematic reviews and meta-analyses depends on the types of studies included in the review. Bibliometric networks were graphically presented using $\mathrm{R}$ packages bibliometrix version 3.0.2 [9] and wordcloud2 version 0.2.1. Geomapping of the evaluated papers by country was completed using the R package rworldmap version 1.3.6 [15]. 


\section{Results}

The results of the search are presented in a flow diagram (Fig. 1). The search yielded 1617 articles, of which 988 were excluded based on the stated exclusion criteria. The study therefore comprises a total of 296 articles on COVID-19 in the field of Dentistry published from February to September 6, 2020 [16]. A complete list of all analyzed articles is given in Online Resource 3. Regarding document types, there were 93 original scientific manuscripts, 168 review papers, five case reports, and 30 short communications published in the English $(n=$ 257), Spanish $(n=24)$, Chinese $(n=9)$, Italian $(n=5)$, and Dutch $(n=1)$ language. Of the 296 published manuscripts, 97\% were Open Access articles.

\section{Authors}

There were 1331 authors, responsible for 1474 authorships, affiliated to 429 institutions from 53 countries. The number of authors per article ranged from 1 to 30 , while the average number of authors per article was 4.5. Of the 296 papers, 26 (8.78\%) were single-authored, 39 (13.18\%) had two authors, $41(13.85 \%)$ three, and the remaining $64.19 \%$ by four or more authors. Baghizadeh Fini M (Tehran University of Medical Sciences, Tehran, Iran; Oklahoma State University, Stillwater, OK, USA) was one of two authors who wrote two singleauthored narrative literature reviews related to oral saliva and a comprehensive guideline for dentists, both published in Oral Oncology [17, 18]. The second author was Harikrishnan P (Teeth "N" Jaws Center, Chennai, India), who also published two narrative reviews on COVID-19 diagnostics, both in the Journal of Craniofacial Surgery [19, 20].

The first five authors who contributed with the largest number of published COVID-19-related research are provided in Online Resource 4. The ranking of authors is based on their total (TA) and adjusted frequency (AF), which reflects multiple-authored articles (i.e., if an article is published by two authors, each receives half a credit). Authors of the analyzed papers are also ranked based on dominance factor (DF), which reflects the proportion of the number of multi-authored
Fig. 1 A flow diagram of the study search and identification. WoS Web of Science Core Collection, KJD Korean Journal Database, RSCI Russian Science Citation Index, SCIELO SciELO Citation Index; Reasons for the exclusion: *, 30 related to SarsCov-1, 18 not dentistry; \#, not original papers; $\$, 257$ not original papers, 9 unavailable

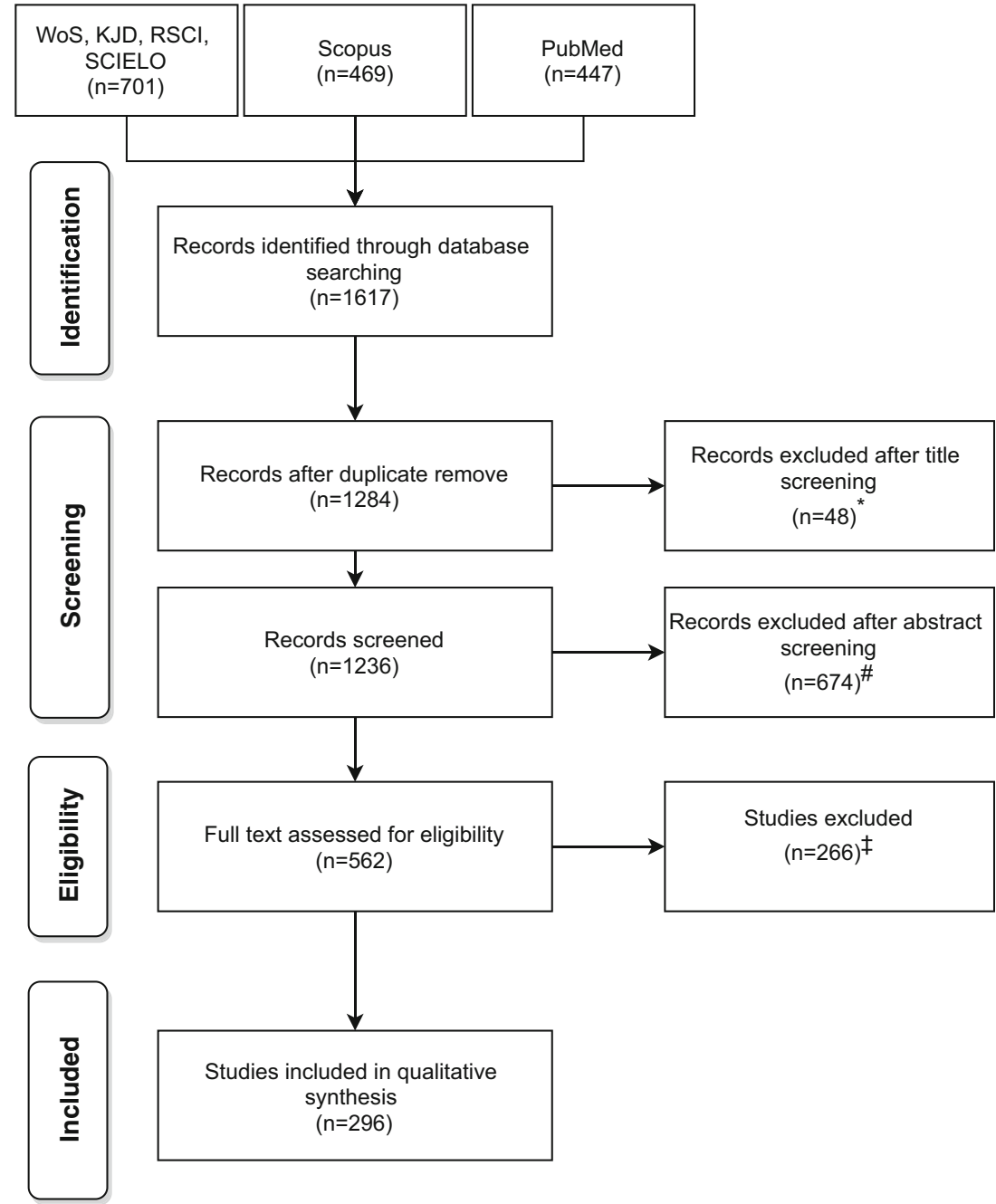


publications of an author as the first author (FA) to the total number of multi-authored publications of an author (MA). As one of the authors appearing in six separate papers $(\mathrm{TA}=6)$, whose fractionalized frequency and DF value were equal to 1.49 and 0.33, respectively, Samaranayake LP (The University of Queensland, Australia) contributed with two systematic literature reviews ( $\mathrm{LoE}$ I and $\mathrm{LoE} \mathrm{V}$ ), three narrative literature reviews, and one short communication. It is interesting to note that the first five authors ranked by the total number of articles (i.e., Brennan PA, Samaranayake LP, Zhou HD, Dziedzic A, and Hua F) (Online Resource 4), who are employed as dentists at different dental schools, could be positively identified as "domain experts," since they have the past publication record related to other viral infections (between 5 and 10 articles, according to PubMed), in addition to the analyzed COVID-19 papers. The scientific collaboration network where nodes are authors and links are co-authorships is given in Online Resource 5, revealing regular study groups and pivotal authors of the COVID-19 research in the field of Dentistry.

\section{Institutions}

Institutions with at least five articles on COVID-19 ordered according to their total frequency of appearance are presented in Online Resource 6. Sichuan University, China, was the most commonly stated institution $(n=22)$, followed by Wuhan University, China ( $n=19)$; the University of São Paulo, Brazil ( $n=14)$; Peking University, China $(n=13)$; the University of Hong Kong, China $(n=13)$; the University of Insubria, Italy $(n=12)$; the University of Toronto, Canada $(n=11)$; and the University of Siena, Italy $(n=10)$. Just over one-third of the analyzed articles $(n=107)$ are singleinstitution papers. Even though Sichuan University, China, was one of the most frequently mentioned institutions, its collaboration was limited to only two institutions, Guangxi Medical University, China, and Texas A\&M College of Dentistry, USA. Wuhan University was also one of the more represented institutions characterized by weak collaboration only with the University of British Columbia, Canada, and the University of Hong Kong. Conversely, Italian institutions, including the University of Milan, the University of Messina, Sapienza University, and University of Naples Federico II, were detected as highly collaborative institutions. The collaboration network among the 250 most frequently appearing institutions can be explored through Online Resource 7, where colors are used to make distinctions between different clusters and the size of the nodes representing institutions indicates the total number of identified COVID-19 articles.

\section{Countries}

Authors from 53 countries, including 17 European, 16 Asian, 12 American, four African, two Oceanian, and two transcontinental countries (Turkey and Egypt), participated in the analyzed articles. Figure 2 shows the contribution of each country within the distribution of dental COVID-19related papers. Based on the country of the corresponding author, more than half of the articles $(n=180,60.8 \%)$ originated from five countries, with nearly half of these articles being from China and Italy $(n=83,46.1 \%), 35(19.4 \%)$ from the UK, 32 (17.8\%) from the USA, and 30 (16.7\%) from Brazil. In addition to the total number of articles (TA), Online Resource 8 presents the number of articles based on the corresponding author's country $(\mathrm{AC})$ and the number of single (SCP) or multi-country papers (MCP). MCPs indicate the number of articles in which at least one co-author is from a country different from the corresponding author's country. Among 52 articles from China, 46 were papers in which the corresponding author was from China. The 35 were singlecountry, while 17 were the result of international collaboration. Most of the COVID-19 papers were single-country papers $(\mathrm{SCP}=241,81.4 \%)$, originating from 36 countries. Of the total number of articles, 42 were the result of cooperation between the two countries, while four and five articles were published as a collaboration of three and four countries, respectively. Cooperation between five, six, and seven countries was noted in three articles only. The greatest level of international collaboration was achieved in one paper only, in which authors from 11 countries (the UK, Netherlands, Latvia, Spain, Italy, Sweden, Hungary, the USA, Germany, France, and Ireland) investigated the immediate response of European Academic Dental Institutions to COVID-19 pandemic and future implications for dental education [21]. The social structure represented on a macro level using the collaboration network among countries is given in Online Resource 9.

\section{Journals}

COVID-19 articles were published in 89 journals, with 48 being indexed on the JCR and having IF. Of the journals without IF, JMIR Public Health and Surveillance was the only title indexed in the MEDLINE, Clarivate Analytics' Science Citation Index Expanded, and Social Sciences Citation Index, while 13 and 8 were listed on the Clarivate Analytics' Emerging Sources Citation Index and MEDLINE, respectively. Nine journals were indexed in both Emerging Sources Citation Index and MEDLINE, while ten titles did not appear on the either internationally significant bibliographic list or citation index. Most of the journals (79.8\%) were dental journals, while the remaining 18 titles belonged to other scientific fields such as Biochemistry and Molecular Biology; Infectious Diseases; General and Internal Medicine; Public, Environmental, and Occupational Health; or Education. Online Resource 10 shows the journals' information regarding the IF of the journal based on the current release of JCRs (JCR@ IF2019), Journal IF 2019 Quartile in WoS subject 
Fig. 2 Participation of each country in the distribution of dental COVID-19 articles

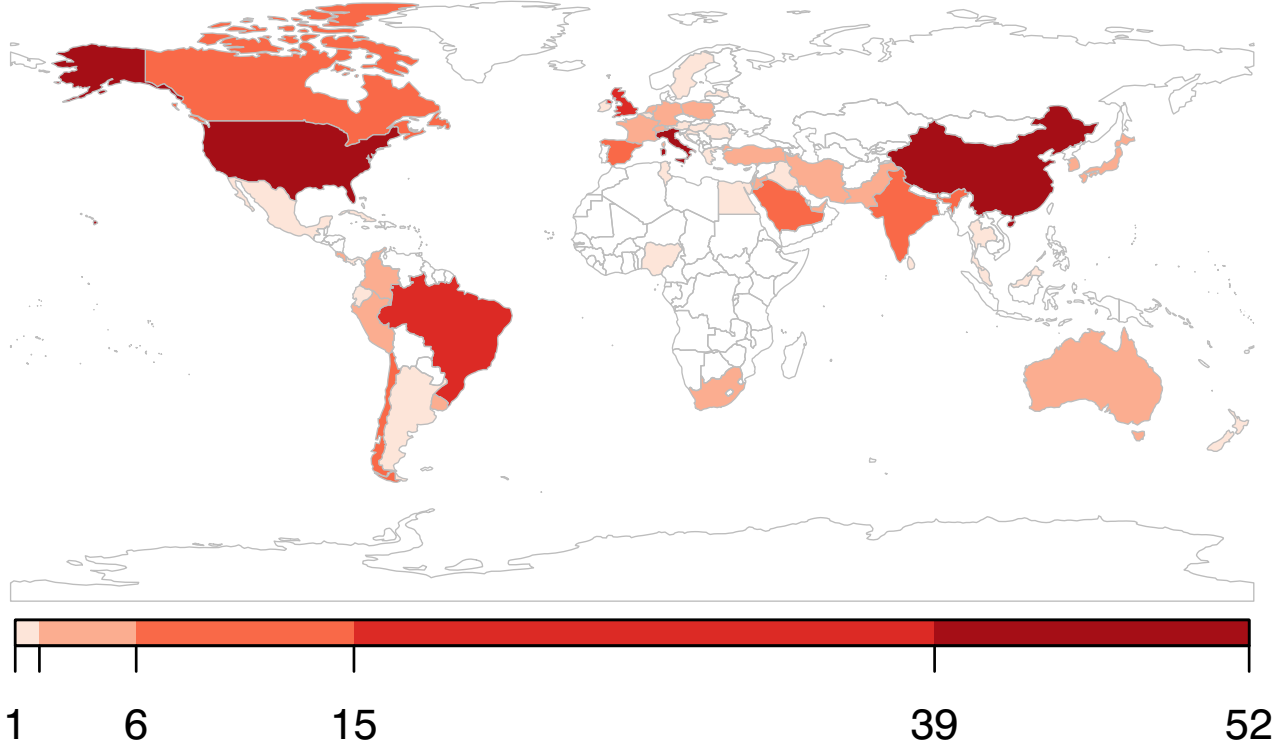

category (QC), the total number of published papers (TA), the total number of received citations (TC), and the average number of citations per article $\left(\mathrm{mTC}_{\mathrm{A}}\right)$.

The largest number of COVID-19 articles was published in Oral Diseases, followed by British Journal of Oral and Maxillofacial Surgery, International Journal of Environmental Research and Public Health, British Dental Journal, International Journal of Odontostomatology, and Brazilian Oral Research. One-third of the articles (33.78\%) were published in these journals, representing the core sources of COVID-19 research. Information on journals that have published five or more COVID-19 articles is presented in Table 1. Articles published in the International Journal of Oral Science, Lancet Infectious Diseases, and Journal of Dental Research were papers with the highest total number of citations. The highest number of average citations per paper was noted from articles published in Lancet Infectious Diseases $\left(\mathrm{mTC}_{\mathrm{A}}=246\right)$ and International Journal of Oral Science $\left(\mathrm{mTC}_{\mathrm{A}}=151.33\right)$. More than half of COVID-19 papers $(n=191,64.53 \%)$ were published in journals listed in JCR lists, mostly in the first (41.36\%) and fourth (31.94\%) JIF Quartiles in subject categories.

\section{Citations}

The analyzed papers received a total of 1280 citations, ranging from 0 to 251 citations per paper. The average number of citations per paper was 4.3. A total of 78 papers had one or more citations, presented in Online Resource 3. The most cited article, dealing with the potential routes of 2019-nCov infection on the mucosa of the oral cavity, was the crosssectional study published in the International Journal of Oral Sciences [22]. Among articles that had ten or more citations, $64.71 \%$ were narrative literature reviews, followed by four cross-sectional, one cohort study, and one short communication. Citation analysis revealed that China was the most influential country with a total of 888 citations, followed by the USA $(\mathrm{TC}=111)$ and Italy $(\mathrm{TC}=85)$. The analyzed articles on COVID-19 published in dental journals $(n=192)$ received a total of 1191 citations and 6.2 citations per article. A total of $45(15.2 \%)$ articles published in non-dental journals received 374 citations, with an average number of citations per paper of 8.3 .

\section{Research topics, study design, and level of scientific evidence}

In the analyzed dataset, a total of 545 authors' keywords and 336 Keywords Plus were identified. In addition to keywords "COVID-19," "Sars-Cov-2," "coronavirus," or "dentistry," the most frequently used authors' keywords were "pandemic," "infection control," "saliva," "oral health," "aerosol," “dental education," "personal protection," "dental practice," or "transmission." The overview of the authors' keywords revealing COVID-19 interests of dental researchers and the frequency of their occurrence is shown in Online Resource 11.

COVID-19 dental research consisted mainly of articles from the field of General Dentistry $(\mathrm{TA}=197)$, dealing mostly with Public Health $(\mathrm{TA}=111)$ or educational $(\mathrm{TA}=11)$ issues, as well as guidelines for dental care improvement (TA = 25). In Oral and Maxillofacial Surgery, the second most represented area $(\mathrm{TA}=32), 21.9 \%$ of articles dealt with the development of guidelines. Besides General Dentistry and Oral and Maxillofacial Surgery, Oral Pathology and Oral Medicine were recognized as some of the well-represented fields of interest as well (TA $=11)$.

In addition to papers focused on several research questions, such as therapy, prevention, diagnosis, prognosis, or etiology 
Table 1 Journals

\begin{tabular}{|c|c|c|c|c|c|}
\hline Sources & JCR® IF2019 & $\mathrm{QC}$ & TA & $\mathrm{TC}^{*}$ & $\mathrm{mTC}_{\mathrm{A}}$ \\
\hline Oral Diseases & 2.613 & $\mathrm{Q} 1^{1}$ & 24 & 56 & 2.33 \\
\hline British Journal of Oral and Maxillofacial Surgery & 1.061 & $\mathrm{Q} 4^{1,2}$ & 22 & 10 & 0.45 \\
\hline International Journal of Environmental Research and Public Health & 2.849 & $\mathrm{Q} 1^{3} / \mathrm{Q} 2^{4}$ & 16 & 47 & 2.94 \\
\hline British Dental Journal & 1.306 & $\mathrm{Q} 4^{1}$ & 14 & 0 & 0 \\
\hline International Journal of Odontostomatology & - & $\mathrm{NA}^{\#}$ & 13 & 11 & 0.85 \\
\hline Brazilian Oral Research & 1.633 & $\mathrm{Q}^{1}$ & 11 & 0 & 0 \\
\hline Pesquisa Brasileira em Odontopediatria e Clínica Integrada & - & $\mathrm{NA}^{\$}$ & 11 & 0 & 0 \\
\hline Journal of Craniofacial Surgery & 0.953 & $\mathrm{Q}^{2}$ & 7 & 0 & 0 \\
\hline Journal of Dental Research & 4.914 & $\mathrm{Q} 1^{1}$ & 7 & 165 & 23.57 \\
\hline Journal of Oral and Maxillofacial Surgery & 2.068 & $\mathrm{Q}^{1}$ & 6 & 4 & 0.67 \\
\hline Oral Oncology & 3.979 & $\mathrm{Q} 1^{1} / \mathrm{Q} 2^{5}$ & 6 & 31 & 5.17 \\
\hline American Journal of Orthodontics and Dentofacial Orthopedics & 1.960 & $\mathrm{Q} 2^{1}$ & 5 & 0 & 0 \\
\hline Brazilian Dental Science & - & $\mathrm{NA}^{\$}$ & 5 & 0 & 0 \\
\hline Chinese Journal of Dental Research & - & $\mathrm{NA}^{\$, \#}$ & 5 & 5 & 1 \\
\hline Clinical Oral Investigations & 2.812 & $\mathrm{Q} 1^{1}$ & 5 & 13 & 2.6 \\
\hline Dental Cadmos & - & $\mathrm{NA}^{\$}$ & 5 & 1 & 0.2 \\
\hline Head and Neck-Journal for the Sciences and Specialties of the Head and Neck & 2.538 & $\mathrm{Q} 1^{6} / \mathrm{Q} 2^{2}$ & 5 & 10 & 2 \\
\hline Journal of Dental Sciences & 1.034 & $\mathrm{Q} 4^{1}$ & 5 & 0 & 0 \\
\hline Odontoestomatologia & - & NA & 5 & 0 & 0 \\
\hline
\end{tabular}

$Q C$ journal impact factor 2019 quartile in subject category, $T A$ the total number of articles, $T C$ the total number of citations for articles, $m T C_{A}$ the average number of citations per article, $N A$ not applicable. WoS subject categories: ${ }^{1}$ DOSM; ${ }^{2}$ Surgery; ${ }^{3}$ Public, Environmental, and Occupational Health;

${ }^{4}$ Environmental Sciences; ${ }^{5}$ Oncology; ${ }^{6}$ Otorhinolaryngology

*The source of citations was Web of Science Core Collection

\# Journal indexed in MEDLINE

\$ Journal indexed in the Clarivate Analytics' Emerging Sources Citation Index

$(\mathrm{TA}=156)$, the most common research issues were prevention $(\mathrm{TA}=43)$ and diagnosis $(\mathrm{TA}=17)$, quality improvement $(\mathrm{TA}=15)$, and therapy $(\mathrm{TA}=14)$. Articles from the field of General Dentistry and Public Health were mostly focused on prevention and diagnosis, while papers related to education dealt generally with the problems of quality improvement.

Regarding the study design, the analyzed research consisted of 160 narrative literature reviews, 73 crosssectional studies, 30 short communications, 11 systematic literature reviews, eight simulation or pilot studies, five observational, four case reports, two case series, two in vitro studies, and one cohort study. The set of examined short communications consisted of papers from the field of General Dentistry (over 70\%), Oral Medicine, and Oral Pathology.

The largest number of articles were within LoE VII (160 narrative and three systematic literature reviews based on guidelines, editorials, letters to the editor, communications, or perspectives), followed by LoE V (80 cross-sectional, observational studies, and case series, as well as two systematic literature reviews based mostly on cross-sectional studies), LoE VI (six case reports), LoE IV (one cohort study and one systematic review based on one randomized controlled trial and observational studies), LoE I (two systematic reviews mostly based on randomized controlled trials), and LoE 0 (three systematic reviews based on in vitro studies, two in vitro, and eight simulation studies).

Table 2 shows a summary of the identified areas of interest, study designs, and levels of scientific evidence. The distribution of analyzed articles according to the study design and journal is presented in Online Resource 12.

\section{Discussion}

Up-to-date scientific research plays a significant role in disease prevention and management, especially at the time of the COVID-19 pandemic, with an accompanying high global mortality rate [23]. Even though the bulk of scientific literature was produced in an incredibly short time frame, an analysis of the research available to base clinical decision-making and treatment support during COVID-19 is required. This bibliometric study is novel in being the first study to identify and assess the characteristics of dental scientific COVID-19 papers published during the initial 8 months of this pandemic. 
Table 2 Study design, field of interest, and the distribution of articles within the pyramid of evidence

\begin{tabular}{|c|c|c|c|}
\hline LoE & Study design & Field/subfield & TA \\
\hline I & Systematic literature review & General Dentistry/Public Health & 2 \\
\hline \multirow{3}{*}{ IV } & & & 2 \\
\hline & Cohort study & General Dentistry & 1 \\
\hline & Systematic literature review & Maxillofacial Surgery & 1 \\
\hline & & & 2 \\
\hline \multirow[t]{15}{*}{$\mathrm{V}$} & Case series & General Dentistry/Oral Medicine & 2 \\
\hline & Cross-sectional study & General Dentistry $^{1}$ & 51 \\
\hline & & Oral and Maxillofacial Surgery & 10 \\
\hline & & Orthodontics & 4 \\
\hline & & Endodontics & 3 \\
\hline & & Dental Traumatology & 1 \\
\hline & & Oral Pathology & 1 \\
\hline & & Oral Radiology & 1 \\
\hline & & Pediatric Dentistry & 1 \\
\hline & & Prosthodontics & 1 \\
\hline & Observational & General Dentistry/Education, Public Health & 4 \\
\hline & & Oral and Maxillofacial Surgery/Guidelines & 1 \\
\hline & Systematic literature review & General Dentistry/Oral Medicine & 1 \\
\hline & & Oral Pathology & 1 \\
\hline & & & 82 \\
\hline VI & Case report & Oral and Maxillofacial Surgery & 4 \\
\hline \multirow{18}{*}{ VII } & & & \\
\hline & Narrative literature review & General Dentistry & 127 \\
\hline & & Oral and Maxillofacial Surgery & 14 \\
\hline & & Oral Medicine & 5 \\
\hline & & Endodontics & 2 \\
\hline & & Orthodontics & 3 \\
\hline & & Dental Anesthesia & 1 \\
\hline & & Forensic Dentistry & 1 \\
\hline & & Microbiology & 1 \\
\hline & & Oral Microbiology & 1 \\
\hline & & Oral Oncology/Guidelines & 1 \\
\hline & & Oral Pathology & 1 \\
\hline & & Oral Radiology & 1 \\
\hline & & Pediatric Dentistry/Guidelines & 1 \\
\hline & & Virusology & 1 \\
\hline & Systematic literature review & General Dentistry/Education & 1 \\
\hline & & Maxillofacial Surgery/Guidelines & 1 \\
\hline & & Prosthodontics & 1 \\
\hline \multirow[t]{3}{*}{0} & In vitro/lab studies & General Dentistry & 1 \\
\hline & & Preventive Dentistry & 1 \\
\hline & Systematic literature review & General Dentistry/Microbiology & 3 \\
\hline \multirow[t]{5}{*}{ Other } & Scientific report/pilot study & General Dentistry & 1 \\
\hline & & Oral Microbiology & 1 \\
\hline & Simulation study & General Dentistry/Public Health & 4 \\
\hline & & Oral and Maxillofacial Surgery & 2 \\
\hline & Short communication & General Dentistry $^{3}$ & 22 \\
\hline
\end{tabular}


Table 2 (continued)

\begin{tabular}{|c|c|c|c|}
\hline LoE & Study design & Field/subfield & TA \\
\hline \multirow{7}{*}{\multicolumn{2}{|c|}{ Total }} & Oral and Maxillofacial Surgery & 2 \\
\hline & & Oral Medicine & 3 \\
\hline & & Oral Pathology & 1 \\
\hline & & Oral Radiology & 1 \\
\hline & & Prosthodontics & 1 \\
\hline & & & 38 \\
\hline & & & 296 \\
\hline \multicolumn{4}{|c|}{$\begin{array}{l}L o E \text { level of evidence; } L o E I \text { systematic review or meta-analysis of randomized controlled clinical trials; } L o E I I \text { randomized controlled clinical trial; } L o E \\
I I I \text { controlled clinical trial; } L o E I V \text { cohort study, case control study, systematic review or meta-analysis of cohort or case control studies, } L o E V \text { cross- } \\
\text { sectional study, case series study, systematic review or meta-analysis of cross-sectional studies, case series or case reports; } L o E V I \text { case report; } L o E V I I \\
\text { narrative literature review, panel and expert opinion, systematic review or meta-analysis of narrative reviews, editorials, guidelines, comments, per- } \\
\text { spectives; } L o E 0 \text { animal research, in vitro/lab studies, systematic review or meta-analysis of animal, in vitro/lab studies; TA the total number of articles; } \\
{ }^{1} \text { subfields (Education, Public Health, Mental Health, Quality of Life), }{ }^{2} \text { subfields (Education, Public Health, Mental Health, Pharmacology, Guidelines, } \\
\text { Pedodontics, Orthodontics), }{ }^{3} \text { subfields (Education, Public Health, Mental Health, Oral Health, Oral Surgery, Guidelines, Gerodontology) }\end{array}$} \\
\hline
\end{tabular}

The examination of 296 scientific articles published until September 2020 reveals which individuals, research groups, institutions, and countries have contributed the most material, as well as identifying which journals have published the greatest volume of COVID-19 dental research, highlighting the principal research issues addressed and their level of scientific evidence.

The principal findings of the study indicate that the majority of the dental scientific COVID-19 articles originate from China and Italy, which is in line with previously conducted bibliometric analysis of COVID-19 literature in the field of Medicine [2, 5]. As a result of the COVID-19 outbreak emerging in China, and subsequently Italy becoming one of the countries dealing with the worst European outbreak, it was expected that the majority of relevant articles were attributed to Chinese and Italian authors and institutions. Given the much higher COVID-19 infection and death rates in the USA, Brazil, and the UK [23], a greater contribution originating from these countries was also expected. Among those countries that published a total of ten or more dental COVID-19 articles, India, Chile, Spain, and Canada were also identified. India, rated as the second highest based on the total number of infected cases and total deaths [23], contributed with a total of 15 articles $(5.1 \%)$. Given the global scale of the problem, a notably low level of international collaboration has been observed. This study found that $81.4 \%$ of the analyzed COVID-19 literature were single-country papers. Among more productive countries that produced six or more articles, the United Arab Emirates and Saudi Arabia were the countries with the highest collaboration rates $(83.3 \%$ and $77.8 \%$ of papers, respectively, were published in cooperation with the authors from other countries). Excluding countries that have published two or fewer papers, Australia and Switzerland were noted as the countries that published four and three articles, respectively, all being the result of international writing collaborations. Notably, high cooperation among the institutions of one country was observed among Italian, British, and Brazilian institutions.

An interesting finding of this study is the impact achieved by the dental COVID-19 articles analyzed, assessed through the total number of citations received. More than half of the papers $(64.5 \%)$ were published in the most influential and relevant journals, whose IF values indicated both the impact of those journals in the subject field and the likely influence that the articles published in them. However, even though based on citation counts, IF of the journal, introduced as a journal performance indicator, proved to be an inadequate tool for measuring article quality [24, 25]. Citation analysis, as one of the best-known bibliometric approaches, is widely used in research output evaluation for assessing research performance or impact of researchers, institutions, articles, and journals. Despite its broad use, the intrinsic value of the citation analysis outcomes is a source of frequent discussion [26, 27], indicating that the number of citations does not necessarily reflect the scientific value of the article. Indeed, for qualitative evaluation and research assessments, the validity and reliability of citation counts require verification and compatibility with peer judgment. Nevertheless, citation counts could not identify a significance that had not been recognized by the scientific community [28, 29]. Although recently published (during 2020), 26.4\% of the analyzed articles have already received at least one citation. The year of publication apparently has an impact on the citation count a paper receives, and older publications will tend to earn more citations than recent ones. However, articles that achieve high impact are usually cited within months of publication and certainly within a year or so [24]. Due to this pattern of immediacy, one of the analyzed cross-sectional studies [22] published in February 2020 was 
already included in the Clarivate Analytics' Essential Science Indicators Highly Cited Papers, according to the second bimonthly of 2020 (updated on July 9, 2020), as a research article published in a dental journal with the highest number of received citations per year. Furthermore, a total of four dental COVID-19 articles [22, 30-32] each received more than 100 citations and can be considered citation "classics" $[33,34]$. A significantly high early citation rate of the original articles favoring COVID-19 research, published in some of the highest ranked medical journals, was also observed [35]. Finally, the total number of citations received undeniably reflects the interest of the international scientific and clinical communities and the urgent need for information valuable in the ongoing struggle with COVID-19, rather than its quality and relevance to clinical practice and research, which remains to be determined over time.

A critical finding of this study relates to the low level of scientific evidence observed in the dental scientific literature on COVID-19. It is apparent that COVID-19 has been the main focus of various researchers and institutions worldwide for the past few months, since 1284 unique publications were identified through the literature search conducted on September 6, 2020. However, only $23.1 \%$ of those were original research articles, which is less than the analyzed production in the medical field [5]. Narrative literature reviews with LoE VII were the most frequent types of study design (54.1\%). Higher-level evidence studies scored with LoE I, IV, or V, such as systematic reviews, cohort, and crosssectional studies, represented only $29.7 \%$ of the sample. However, our analysis revealed that only two investigations $(0.67 \%)$ were systematic literature reviews of randomized controlled trials with the highest LoE I [36, 37]. Both reviews investigated the prevention of COVID-19 infection (e.g., reduction of aerosolized microbes in dental practice or effectiveness of respiratory protective equipment (RPE), respectively). In this regard, Samaranayake et al. [37] concluded that RPE is effective as a barrier protection against aerosolized microbes in healthcare settings and that their filtration efficacy is compromised by the inhalant particle size, airflow dynamics, mask-fit factor, period of wear, "wetness" of the masks, and their fabrication quality. In addition, Koletsi et al. [36] revealed that the use of preprocedural mouth rinse with preferably tempered chlorhexidine $0.2 \%$ was the most effective strategy for the reduction of aerosol-related bacterial load in dental practice. The majority of analyzed systematic reviews, based on in vitro studies, narrative reviews, editorials, guidelines, comments, perspectives, and cohort or cross-sectional studies, were graded as studies with LoE 0 , VII, V, or IV. Among investigated COVID-19 articles, a notable portion consisted of cross-sectional studies (24.7\%), conducted mainly in the field of General Dentistry and related to Public Health or educational issues, as well as in the field of Oral and Maxillofacial Surgery, Orthodontics, and Endodontics.
There were no randomized clinical trials in the analyzed set of articles. These findings imply that the overall LoE in the COVID-19 dental literature is disappointingly low, not providing data relevant for the evidence-based decision-making process, which unfortunately is consistent with data found in other medical fields $[2,5,38]$. For instance, the overall quality of evidence of COVID-19 articles, published in the three highest ranked scientific medical journals during the same period (i.e., The New England Journal of Medicine, The Lancet, and The Journal of the American Medical Association), is found to be below the quality average of these journals [35]. Therefore, more investigations in the future should be performed as randomized clinical trials, so a clearer picture of the status of COVID-19 infection in different fields of dentistry emerges.

Based on the analysis of keywords and noted fields of interest, three main research themes of dental COVID-19 papers were highlighted. This study identified top keywords that appeared in the scientific literature and demonstrated how they appeared across several studies. One of the significant interests among investigations that have been recently raised was the potential use of saliva and other oral fluids as promising samples of choice for COVID-19 testing. From the beginning of the pandemic, a quantitative real-time polymerase chain reaction (qRT-PCR) analysis on pharyngeal or respiratory tract swabs was considered the gold standard for the detection of COVID-19 [39]. This approach is relatively invasive, induces coughing and occasionally causes bleeding, and may potentially be a source of viral transmission [30]. However, previous studies promoted the idea that the socalled posterior oropharyngeal saliva (i.e., sputum or oropharyngeal secretions) is also a reliable source of COVID-19 [40]. In contrast to nasopharyngeal swabs, collecting sputum is less invasive and can be performed by the patient. On the other hand, it should be performed before tooth brushing and breakfast, and in some cases, it may not be possible to provide sputum $[30,41]$. Several studies revealed the presence of the virus in the salivary specimens of patients with previously laboratory-confirmed COVID-19 [30, 42]. Bajaj et al. [43] presented a brief review of the diagnostic potential of saliva as a vehicle for COVID-19 testing. In general, potential salivary diagnostic tests, recognized on the international and national level, may potentially be more beneficial as they are less invasive, cheaper, and easy to perform. However, future investigations are needed to standardize the method for the collection of saliva, implement the use of appropriate assays, and outline processing methods.

Dental education in the COVID-19 era is another frequently discussed topic. One of the most important challenges in dental education during this pandemic was to maintain the balance between continuing the education process for dental students and eradication of the virus. Practically, the curriculum of all dental schools worldwide is based on lectures, 
simulation laboratory courses, and clinical skills training. Included articles revealed that clinical activities at least for some time in most dental schools were restricted only to emergency cases [44-46]. Therefore, in order to ensure social distance, all lectures were switched to online courses using the available applications. In addition, simulation laboratory courses were postponed or presented as a video demonstration of simulation. However, due to the necessity of hands-on training for some preclinical laboratory courses, the authors suggested the possibility for the development of easy-to-use virtual reality haptic devices. The principal educational problem for undergraduate studies was that clinical training courses were completely suspended. On the other hand, clinical training for postgraduate students was restricted only to emergency cases. However, a survey of Huntley et al. [47] revealed that residents' major concerns are directed toward the lack of clinical operative experience. Despite the possibilities of virtual education, Al-Taweel et al. [48] reported that dental students demonstrated low-moderate satisfaction with technology-based learning (such as visual media, digitalized content, interactive video tools, graphs, or web-based interaction) and the quality of material presented to them.

Thirty-seven of 296 included studies dealt with guidelines for the prevention of COVID-19 transmission in dental practice. Most of them were related to general dental practice, although several guidelines were related to specific dental branches (Oral and Maxillofacial Surgery, Oral Medicine, Orthodontics, Endodontics, etc.). All of them suggested how to act during the patient triage, patients' entrance into the practice, dental treatment, and postoperative management. A special focus was directed to the appropriate use of personal protective equipment for dental practitioners, limitation of aerosol-producing procedures, and adequate cleaning of potentially contaminated surfaces. Although the guidelines that were presented are quite useful in everyday clinical practice, their potential limitation is that these are often geographically specific and may not be of international relevance or importance. Clinical COVID-19 guidelines produced in the early stage of the pandemic demonstrated methodological weaknesses, especially in the rigor of their development, and poor overall quality [49]. Perhaps it is irrational to expect strongly evidenced guidelines for a recently emerged disease when no or only weak evidence is available.

Despite the massive publication effort and the existing information overload of COVID-19 research, the findings of this study demonstrated a worrying trend of low-evidenced COVID-19 literature. A higher prevalence of narrative literature reviews and guidance articles, as well as a lower prevalence of experimental research, indicate a considerable gap in a current COVID-19 knowledge base. Studies offering weak evidence, old findings, or data that cannot be generalized may lead to the development of improper procedures and inaccurate and misleading translation into clinical practice.

\section{Strengths}

To the best of the authors' knowledge, this is the first report of the characteristics of the scientific literature addressing COVID-19 from a dental research perspective. Furthermore, besides descriptive analysis, this study offers insightful data on the extent, type, and LoE of COVID-19 scientific output and identification of well-resourced areas of study in Dentistry. Also, a complete metadata set of 296 analyzed COVID-19 articles is shared and reusable in future investigations related to COVID-19 dental scientific production.

\section{Limitations}

Although the most comprehensive international (WoS, Scopus, and PubMed) and national (Korean Journal Database, Russian Science Citation Index, SciELO Citation Index) databases have been used for retrieval of scientific medical literature, they do not include journals of national importance indexed, for instance, in Chinese databases, in which relevant papers may have been published. Additionally, the most recent articles that were accepted but not published at the time of the literature search were not included in this study, so the total number of relevant papers is expected to be much higher. Finally, editorials are excluded from this study, even though they present original and significant material that may contain as much scientific information as short communications. Despite these limitations, we believe that the data presented in this study still provide significant insight into the scope and type of the large body of COVID-19 dental literature.

\section{Conclusion}

These findings provide a relatively objective reference for peer scientists, editors and publishers, clinicians, healthcare providers, and policymakers to dental research trends and publishing patterns related to the current COVID-19 pandemic. With the quite low scientific evidence found in COVID-19 dental literature, not providing data relevant for the evidencebased decision-making process, future investigations are needed to obtain more precise knowledge of COVID-19 infection status in different fields of dentistry.

Supplementary Information The online version contains supplementary material available at https://doi.org/10.1007/s00784-021-03916-6.

\section{Declarations}

Ethics approval This article does not contain any studies with human participants or animals performed by any of the authors. 
Informed consent For this type of study, formal consent is not required.

Conflict of interest The authors declare no competing interests.

\section{References}

1. Ioannidis JPA, Thombs BD (2019) A user's guide to inflated and manipulated impact factors. Eur J Clin Investig 49:e13151. https:// doi.org/10.1111/eci.13151

2. Di Girolamo N, Meursinge Reynders R (2020) Characteristics of scientific articles on COVID-19 published during the initial 3 months of the pandemic. Scientometrics 125:795-812. https://doi. org/10.1007/s11192-020-03632-0

3. Soltani P, Patini R (2020) Retracted COVID-19 articles: a sideeffect of the hot race to publication. Scientometrics 125:819-822. https://doi.org/10.1007/s11192-020-03661-9

4. Hossain MM (2020) Current status of global research on novel coronavirus disease (COVID-19): a bibliometric analysis and knowledge mapping. F1000Res 9:374. https://doi.org/10.12688/ f1000research.23690.1

5. Chahrour M, Assi S, Bejjani M et al A Bibliometric analysis of COVID-19 research activity: a call for increased output. Cureus 12. https://doi.org/10.7759/cureus. 7357

6. Lou J, Tian S-J, Niu S-M et al (2020) Coronavirus disease 2019: a bibliometric analysis and review. Eur Rev Med Pharmacol Sci 24: 3411-3421. https://doi.org/10.26355/eurrev_202003_20712

7. RStudio Team (2020) RStudio: integrated development for R. RStudio, PBC, Boston

8. Ackley BJ, Ladwig GB, Swan BA, Tucker SJ (2008) Evidencebased nursing care guidelines: medical-surgical interventions. Mosby Elsevier, St. Louis

9. Aria M, Cuccurullo C (2017) bibliometrix: An R-tool for comprehensive science mapping analysis. J Informetr 11:959-975. https:// doi.org/10.1016/j.joi.2017.08.007

10. Abramo G, D'Angelo CA, Rosati F (2013) The importance of accounting for the number of co-authors and their order when assessing research performance at the individual level in the life sciences. J Informetr 7:198-208. https://doi.org/10.1016/j.joi. 2012.11.003

11. Kumar S, Kumar S (2008) Collaboration in research productivity in oil seed research institutes of India. In: Kretschmer H, Havemann F (eds) Proceedings of Fourth International Conference on Webometrics, Informetrics and Scientometrics. Humboldt Universitat zu Berlin, Institute for Library and Information Science (IBI), Berlin, pp 1-16

12. Glänzel W, Schubert A (2005) Analysing scientific networks through co-authorship. In: Moed HF, Glänzel W, Schmoch U (eds) Handbook of Quantitative Science and Technology Research: The Use of Publication and Patent Statistics in Studies of S\&T Systems. Springer Netherlands, Dordrecht, pp 257-276

13. Kamada T, Kawai S (1989) An algorithm for drawing general undirected graphs. Inf Process Lett 31:7-15. https://doi.org/10.1016/ 0020-0190(89)90102-6

14. Blondel VD, Guillaume J-L, Lambiotte R, Lefebvre E (2008) Fast unfolding of communities in large networks. J Stat Mech 2008: P10008. https://doi.org/10.1088/1742-5468/2008/10/P10008

15. South A (2011) rworldmap: a new R package for mapping global data. R J 3:35-43

16. Jacimovic J, Jakovljevic A (2020) Dental scientific literature on COVID-19. Mendeley Data, V1. https://doi.org/10.17632/ f989pchhw3.1
17. Fini MB (2020) What dentists need to know about COVID-19. Oral Oncol 105:104741. https://doi.org/10.1016/j.oraloncology.2020. 104741

18. Fini MB (2020) Oral saliva and COVID-19. Oral Oncol 108: 104821. https://doi.org/10.1016/j.oraloncology.2020.104821

19. Harikrishnan P (2020) Gustatory dysfunction as an early symptom in COVID-19 screening. J Craniofac Surg 31:e656-e658. https:// doi.org/10.1097/SCS.0000000000006797

20. Harikrishnan $P$ (2020) Saliva as a potential diagnostic specimen for COVID-19 testing. J Craniofac Surg 31:e653-e655. https://doi.org/ 10.1097/SCS.0000000000006724

21. Quinn B, Field J, Gorter R, Akota I, Manzanares MC, Paganelli C, Davies J, Dixon J, Gabor G, Amaral Mendes R, Hahn P, Vital S, O'Brien J, Murphy D, Tubert-Jeannin S (2020) COVID-19: The immediate response of European academic dental institutions and future implications for dental education. Eur J Dent Educ 24:811814. https://doi.org/10.1111/eje. 12542

22. Xu H, Zhong L, Deng J, Peng J, Dan H, Zeng X, Li T, Chen Q (2020) High expression of ACE2 receptor of 2019-nCoV on the epithelial cells of oral mucosa. Int J Oral Sci 12:1-5. https://doi.org/ 10.1038/s41368-020-0074-x

23. WHO (2020) Coronavirus disease (COVID-19) - World Health Organization. https://www.who.int/emergencies/diseases/novelcoronavirus-2019.

24. Garfield E (2006) The history and meaning of the journal impact factor. JAMA 295:90-93. https://doi.org/10.1001/jama.295.1.90

25. Casadevall A, Fang FC (2015) Impacted Science: Impact Is Not Importance. mBio 6:e01515. https://doi.org/10.1128/mBio.0159315

26. Whitehouse GH (2001) Citation rates and impact factors: should they matter? Br J Radiol 74:1-3. https://doi.org/10.1259/bjr.74.877. 740001

27. Seglen PO (1998) Citation rates and journal impact factors are not suitable for evaluation of research. Acta Orthop Scand 69:224-229. https://doi.org/10.3109/17453679809000920

28. Garfield E (1979) Citation indexing - its theory and application in science, technology, and humanities. Wiley, New York

29. Fardi A, Kodonas K, Lillis T, Veis A (2017) Top-cited articles in implant dentistry. Int J Oral Maxillofac Implants 32:555-564. https://doi.org/10.11607/jomi.5331

30. To KK-W, Tsang OT-Y, Leung W-S et al (2020) Temporal profiles of viral load in posterior oropharyngeal saliva samples and serum antibody responses during infection by SARS-CoV-2: an observational cohort study. Lancet Infect Dis 20:565-574. https://doi.org/ 10.1016/S1473-3099(20)30196-1

31. Peng X, Xu X, Li Y, Cheng L, Zhou X, Ren B (2020) Transmission routes of 2019-nCoV and controls in dental practice. Int J Oral Sci 12:9. https://doi.org/10.1038/s41368-020-0075-9

32. Meng L, Hua F, Bian Z (2020) Coronavirus disease 2019 (COVID19): emerging and future challenges for dental and oral medicine. J Dent Res 99:481-487. https://doi.org/10.1177/0022034520914246

33. Andersen J, Belmont J, Cho CT (2006) Journal impact factor in the era of expanding literature. J Microbiol Immunol Infect 39:436-443

34. Feijoo JF, Limeres J, Fernández-Varela M, Ramos I, Diz P (2014) The 100 most cited articles in dentistry. Clin Oral Investig 18:699 706. https://doi.org/10.1007/s00784-013-1017-0

35. Zdravkovic M, Berger-Estilita J, Zdravkovic B, Berger D (2020) Scientific quality of COVID-19 and SARS CoV-2 publications in the highest impact medical journals during the early phase of the pandemic: a case control study. PLoS One 15:e0241826. https:// doi.org/10.1371/journal.pone.0241826

36. Koletsi D, Belibasakis GN, Eliades T (2020) Interventions to reduce aerosolized microbes in dental practice: a systematic review with network meta-analysis of randomized controlled trials. J Dent Res 99:1228-1238. https://doi.org/10.1177/0022034520943574 
37. Samaranayake LP, Fakhruddin KS, Ngo HC, Chang JWW, Panduwawala C (2020) The effectiveness and efficacy of respiratory protective equipment (RPE) in dentistry and other health care settings: a systematic review. Acta Odontol Scand 78:626-639. https://doi.org/10.1080/00016357.2020.1810769

38. Zocchi J, Pietrobon G, Moretto S, Bonsembiante A, Mazzola F, Petruzzi G, Iocca O, Pichi B, Ansarin M, Pellini R (2020) Literature in the time of COVID-19: the "phase two.". Oral Oncol 109:104837. https://doi.org/10.1016/j.oraloncology.2020.104837

39. Wang Y, Kang H, Liu X, Tong Z (2020) Combination of RT-qPCR testing and clinical features for diagnosis of COVID-19 facilitates management of SARS-CoV-2 outbreak. J Med Virol 92:538-539. https://doi.org/10.1002/jmv.25721

40. Braz-Silva PH, Pallos D, Giannecchini S, To KK-W (2020) SARSCoV-2: what can saliva tell us? Oral Dis 27:746-747. https://doi. org/10.1111/odi.13365

41. Wölfel R, Corman VM, Guggemos W, Seilmaier M, Zange S, Müller MA, Niemeyer D, Jones TC, Vollmar P, Rothe C, Hoelscher M, Bleicker T, Brünink S, Schneider J, Ehmann R, Zwirglmaier K, Drosten C, Wendtner C (2020) Virological assessment of hospitalized patients with COVID-2019. Nature 581:465469. https://doi.org/10.1038/s41586-020-2196-x

42. Azzi L, Carcano G, Dalla Gasperina D, Sessa F, Maurino V, Baj A (2020) Two cases of COVID-19 with positive salivary and negative pharyngeal or respiratory swabs at hospital discharge: a rising concern. Oral Dis 27:707-709. https://doi.org/10.1111/odi.13368

43. Bajaj N, Granwehr BP, Hanna EY, Chambers MS (2020) Salivary detection of SARS-CoV-2 (COVID-19) and implications for oral health-care providers. Head Neck 42:1543-1547. https://doi.org/ 10.1002/hed.26322

44. Machado RA, Bonan PRF, Perez DE d C, Martelli JÚnior H (2020) COVID-19 pandemic and the impact on dental education: discussing current and future perspectives. Braz Oral Res 34: e083. https://doi.org/10.1590/1807-3107bor-2020.vol34.0083

45. Iyer P, Aziz K, Ojcius DM (2020) Impact of COVID-19 on dental education in the United States. J Dent Educ 84:718-722. https://doi. org/10.1002/jdd. 12163

46. Spanemberg JC, Simões CC, Cardoso JA (2020) The impacts of the COVID-19 pandemic on the teaching of dentistry in Brazil. J Dent Educ 84:1185-1187. https://doi.org/10.1002/jdd.12364

47. Huntley RE, Ludwig DC, Dillon JK (2020) Early effects of COVID-19 on oral and maxillofacial surgery residency trainingresults from a national survey. J Oral Maxillofac Surg 78:12571267. https://doi.org/10.1016/j.joms.2020.05.02648

48. Al-Taweel FB, Abdulkareem AA, Gul SS, Alshami ML (2020) Evaluation of technology-based learning by dental students during the pandemic outbreak of coronavirus disease 2019. Eur J Dent Educ 25:183-190. https://doi.org/10.1111/eje.12589

49. Dagens A, Sigfrid L, Cai E, Lipworth S, Cheng V, Harris E, Bannister P, Rigby I, Horby P (2020) Scope, quality, and inclusivity of clinical guidelines produced early in the covid-19 pandemic: rapid review. BMJ 369:m1936. https://doi.org/10.1136/bmj.m1936

Publisher's note Springer Nature remains neutral with regard to jurisdictional claims in published maps and institutional affiliations. 